\title{
Prevalence of Goiter and Associated Factors among Women of Reproductive Age Group in Demba Goffa Woreda, Gamo Goffa Zone, Southwest Ethiopia: A Community-based Cross-sectional Study
}

\section{Befikaduwa Zekarias}

Mizan Tepi University

Frehiwot Mesfin

Haramaya University

Bezatu Mengiste

Haramaya University

Adane Tesfaye

Dilla University

Lemma Getacher ( $\square$ lemmagetacher@gmail.com )

Debre Berhan University https://orcid.org/0000-0002-9237-117X

\section{Research Article}

Keywords: Goiter, lodine Deficiency Disorders, lodine, Demba Woreda, Ethiopia

Posted Date: October 20th, 2020

DOI: https://doi.org/10.21203/rs.3.rs-94175/v1

License: (c) (i) This work is licensed under a Creative Commons Attribution 4.0 International License.

Read Full License 


\section{Prevalence of Goiter and Associated Factors among Women of Reproductive Age Group in Demba}

\section{Goffa Woreda, Gamo Goffa Zone, Southwest Ethiopia: A Community-based Cross-sectional Study}

\section{Befikaduwa Zekarias ${ }^{1}$, Frehiwot Mesfin², Bezatu Mengiste ${ }^{2}$, Adane Tesfaye ${ }^{3}$ and Lemma Getacher ${ }^{*}$}

${ }^{1}$ Department of Public Health, College of Health and Medical Science, Mizan Tepi University, Mizan Tepi, Ethiopia

${ }^{2}$ Department of Public Health, College of Health and Medical Science, Haramaya University, Haramaya, Ethiopia

${ }^{3}$ Department of Nutrition, School of Public Health, College of Medicine and Health Science, Dilla University, Dilla, Ethiopia

${ }^{4}$ Department of Public Health, College of Health Science, Institute of Medicine and Health Science, Debre Berhan University, Debre Berhan, Ethiopia

${ }^{*}$ Corresponding author E-mail: lemmagetacher@gmail.com

\section{Abstract}

Background: Iodine deficiency disorder is a major public health problem in Ethiopia that is more common in women of reproductive age. However, it is not well addressed and there is a lack of information on its prevalence and associated factors in women of reproductive age group. Therefore, the objective of this study was to assess goiter prevalence and associated factors among women of reproductive age in the Demba Goffa Woreda, Gamo Goffa Zone, and Southwest Ethiopia.

Methods: A community-based cross-sectional study was used among 584 randomly selected women in the reproductive age group from February 05 to April 20, 2016. A simple random sampling technique was used to select the study Kebeles, and a systematic random sampling technique was used to select the study samples. Data were collected through a pretested questionnaire, and the goiter examination was done clinically for each participant. The collected data were coded and entered into a computer for statistical analysis using Epi-data version 3.2 and analyzed using SPSS version 20. Variables with a P-value $\leq 0.25$ in bivariate logistic regression analysis were entered into multivariate logistic regression analysis, and 
finally, variables with a P-value $<0.05$ in multivariate logistic regression were considered significantly associated with the dependent variable.

Results: The total goiter rate was $43 \%, 95 \% \mathrm{CI}=(39.2-46.9)$. Cassava consumption $(\mathrm{AOR}: 2.02,95 \% \mathrm{CI}$ : (1.03-4)), salt wash before use (AOR: 3.14, 95\% CI: (1.1-11.3)), salt use after > 2 months of purchase (AOR: 11, 95\% CI; (5-26)), family history of goiter (AOR: 4.6, 95\% CI: (1.4-15.8)), and poor knowledge of iodized salt (AOR: 2.7, 95\% CI (1.4-5.5)) were significant factors associated with goiter.

Conclusion: Iodine deficiency was found to be severe in women of reproductive age in the study area. This showed that women of reproductive age, especially during pregnancy, are exposed to iodine deficiency and its adverse effects at delivery. Thus, they need urgent supplementation with iodine, improved access to foods rich in iodine and intake of iodized salt. Additionally, health education should focus on the importance of iodized salt, the proper method of use and the prevention of iodine deficiency, which are highly recommended to minimize the problem.

Keywords: Goiter, Iodine Deficiency Disorders, Demba Woreda

\section{Background}

Iodine, a trace element found in soil, is a key component of thyroid hormones involved in regulating the body's metabolic processes related to normal growth and development of most organs, especially the brain [1-3]. It is an essential micronutrient that our body cannot produce. Our body gates this micronutrient from the environment through food and drinks. In addition, it cannot be stored in the body for a long period of time; therefore, a daily supply is required [2].

Lack of adequate iodine in women prior to and during pregnancy could result in giving birth to children with irreversible brain damage. In addition, other complications during pregnancy are abortion, stillbirth, congenital defects, psychomotor defects, cretinism, mental retardation, paraplegia, deaf mutism, dwarfism, infant mortality, neonatal goiter, and hypothyroidism, which reduce school performance in children and 
brings goiters [3]. Iodine deficiency is the world's single most significant cause of preventable and irreversible brain damage and mental retardation $[3,4]$.

The most visible effect of iodine deficiency is goiter, a condition defined when "each of the lateral lobes of the thyroid gland is larger than the terminal phalanxes of the thumb of the person examined, which is an indicator of chronic iodine deficiency [5]. Goiter can be used as a baseline assessment of a region's iodine status and as a sensitive long-term indicator for the success of an iodine programmer. Goiter due to iodine deficiency is measured through indictors, including urinary iodine concentration (UI), total goiter prevalence (TGP) and through the rates of coverage of adequately iodized salt ( $>15 \mathrm{ppm})$ in households and goiter prevalence among school children and pregnant mothers used to show the overall prevalence of goiter [6].

It has been reported that 2.2 billion people (38\% of the world's population) live in areas with iodine deficiency and are at risk of its complications. Goiter due to iodine deficiency (ID) is the major public health problem in several areas of world. Its prevalence in females in the reproductive age group is very high, ranging from $34.5 \%$ to $37 \%$ [7]. A study performed in India Kashmiri showed that the prevalence of grade one goiter was $12.68 \%$, and the prevalence was high in the 20-24year age group [8]. Similarly, another study in Pakistan Bahawalpur district showed that the prevalence of goiter in females was grade $0=35.7 \%$, grade $1=42.85 \%$ and grade $2=21.42 \%$, and the prevalence decreased as age increased [9].

Iodine deficiency is as high as $42 \%$ in Africa. Approximately 350 million Africans remain under at risk of IDD, which is a quarter of the global burden of iodine deficiency (10). From 40 African countries that had Urinary Iodine Concentration (UIC), fifteen countries (38\%) were iodine deficient, 22 countries (55\%) had adequate iodine nutrition, and three countries (8\%) had above the recommended intake. In Africa, the largest burden of ID, because of the large population size, was in Ethiopia, Sudan, Algeria, Morocco, Gahanna, and Mozambique [11].

In Ethiopia, more than 35 million people are at risk of iodine deficiency, and approximately 28 million people have goiter. This makes it a major public health problem in the country [12]. Endemic and nonendemic areas have high goiter rates. From a global perspective, Ethiopia ranks $6^{\text {th }}$ among 13 "make or break countries", which significantly adds to the global high iodine deficiency disorders (IDD) burden. It 
is one of the nations with the lowest consumption of iodized salt $(15 \%)$ and the highest goiter prevalence (36\%) in Africa. Ethiopia has approximately 66 million persons "unprotected from iodine deficiency" [4]. Across the world, iodized salt and seafood are generally the major dietary sources of this nutrient [13]. The regular use of iodized salt constitutes simple prevention. "The amount of iodine needed over a lifetime is equivalent to a mere teaspoonful," Says Dr Iqbal Kabir, a nutrition expert from UNICEF. "Prevention costs less than a cup of tea." However, in Ethiopia, the preventive approach is so weak that only $15 \%$ of households use iodized salt. The percentage is higher in urban areas $(23 \%)$ than in rural areas (14\%). Even the coverage of iodized salt supplementation differs from region to region in Ethiopia; for example, the proportion of women living in a household with iodized salt is nearly three times higher in the BenshangulGumuz region (45\%) than the national average $(15 \%)[4,14]$.

Regarding the causes of goiter, as different studies show, non-iodized salt and insufficient iodine intake due to inadequate contents of iodine in sea foods, dairy products and iodized salt are the major causes of IDD. In addition, family history of goiter, depleted soil of iodine, crops grown on iodine-depleted soil, subsistence agriculture, exposure to goitrogenic food and substances, excessive cabbage consumption, poor time of salt storage ( $>2$ months), poor knowledge and practice towards iodized salt and IDD prevention mechanisms and socio-demographic factors such as age, occupation, income, family size, educational status of females and their husband and occupation of women's and their husband are the major associated factors of goiter among the WRA group $[5,12,14,15,17,18,21,22]$.

Although many studies show that IDD is the major public health problem in the country and that the problem is more common in females than in males, there is a dearth of information on the problem in women of child-bearing age. Most of the previous studies show only the prevalence of goiter among pregnant women, school children and primary school girls. The previous studies are also inconsistent and do not indicate this problem in all WRA groups in a single study. In addition, these studies do not include the prevalence and factors of goiter among WRAs, including lactating mothers and adolescent girls, in a single study. Another important reason for this study is to see the spatial difference in the prevalence of goiter among these populations. Moreover, the study area, Demba Goffa Woreda, is one of the endemic areas of goiter prevalence in the country. Therefore, the main objective of this study was to assess the 
prevalence and factors associated with goiter in women of reproductive age in Demba Goffa Woreda.

\section{Methods and Materials}

\subsection{Study Setting and Design}

A Community-based cross-sectional quantitative study method was applied from February 05- April 20, 2016 in Demba Goffa woreda. The study was carried out on four randomly selected kebeles in Demba Goffa Woreda, which is one of the 15 Woreda of the Gamo Goffa Zone in SNNPRS. The Woreda is located $510 \mathrm{~km}$ south of Addis Ababa. The Woreda divided into 38 rural Kebeles. The capital town of the Woreda is Sawla. The study area has three agro-climatic zones: highland altitude /Dega/ (7.8\%), mid-altitude /Woina Dega/ (15.8\%) and lowland altitude /Kola/ (76.4\%). The population of the Woreda is 99,891 (male: 49446 and female: 50445 and from this 19819 are women in the reproductive age group). Maize and teff from cereals, sweet potatoes and cassava from roots, moringa ("Haleko") and cabbage from vegetables were the major food types in this area. Cassava and cabbage are consumed in the great majority of households being processed, either alone or mixed with other cereal foods [9].

\subsection{Study Population, Sample Size and Sampling Procedures}

Females in the child bearing age group who were apparently healthy and willing to participate in the study were included in the study and severely ill to respond and had difficulty in speech or listing were excluded. To determine the sample sizes for the prevalence of goiter, single population proportion and with the following assumptions to optimum sample size: $\mathrm{p}=$ prevalence of goiter to be $35.8 \%$ (95\% CI 34.5-37.1) $[5], Z=$ reliability coefficient $=1.96, d=5 \%$ margin of error and, design effect $(\mathrm{DE})=1.5$, non-response rate $=10 \%$ was used. Accordingly, the sample size was 584 .

On the other hand, to determine the sample size for associated factors, a double population formula was used based on the following assumption: $95 \%$ confidence level, power $80 \%$, ratio 1:1 and using the variable from a previous study, goiter $(\mathrm{P} 1=$ proportion for women with every day cassava intake (exposed), $73 \%,(\mathrm{P} 2=$ proportion of women who never consume cassava) (unexposed), 27\%, which gives the largest sample size of among all other variables [2] using Epi info version 7. Then, the calculated sample size was 
44. Considering the $10 \%$ non-response and design effect, the sample size became 74 . Therefore, comparing the two samples sizes, the maximum sample size (584) was taken as the final sample size of the study.

With regard to the sampling procedures, there were 38 Kebeles in Demba Goffa Woreda. Of these, Lamaya Tsala, Banda, Zanga and Yala Kebeles were randomly selected using lottery method. After calculating proportional sample size, the sample size was allocated proportionally to each kebele. Accordingly, the samples were 182 from Layma Thala, 113 from Banda, 186 from Zanga, and 103 fromYala Kebeles. Then, the households from selected Kebeles were selected by systematic random sampling method $\left(\mathrm{N} / \mathrm{n}=\mathrm{n}_{\mathrm{th}}\right)$. The first household in each Kebele was selected randomly by lottery method and the rest were every respected $\mathrm{n}_{\mathrm{th}}$ until the total sample size was achieved.

\subsection{Data Collection Instruments and Procedures}

A pretested and structured interviewer administered questionnaire that developed after reviewing different studies was used to collect the data $[2-5,8,12,14-16,21,22]$. The questionnaire was initially prepared in English, translated into the local language (Amharic and Gofigna) and translated back to English to maintain its consistency. The questionnaires were grouped and arranged as sociodemographic characteristic of the respondents, quarries to assess contributing factors of goiter, quarries to assess knowledge and practice of the respondents towards utilization of iodized salt and IDD prevention and evaluation and grading of goiter.

The data collectors of the study were three health officers and three girls who were complete $10^{\text {th }}$ grades were recruited who had previous data collection experience. The health officers were trained by medical doctors on how to perform the standardized physical examination of goiters, and then they underwent goiter examination. The girls who completed the $10^{\text {th }}$ grades were trained by a principal investigator on how to interview the respondents for two days. Two supervisors, the medical doctors and the principal investigator, supervised the data collection. The collected data were checked by the supervisors and principal investigator on a daily basis for any incompleteness and possible corrections were made.

Regarding the data collection of the dietary characterstics of the respondents, there were questions used to assess the diets of the respondents. These questions assess the eating pattern of different food groups, such as meat/milk/fish, cassava, cabbage, and other important diets including the frequency of these foods in a 
day, week and month.

The knowledge of respondents towards the prevention of IDD were assessed using six questions that assess the awareness of the participants on sources of information, causes of goiter, methods of prevention of goiter, utilization of iodized salt and its storage.

Goiter examination was performed for all participants. To control errors and maximize the data quality, careful training and experienced observation was performed. To minimize inter-individual variability using inspection and palpation, goiter grading was performed as per the recommendation of the 2014 WHO/UNICEF/ICCID. The classification scheme was as follows: Grade 0: no goiter (neither visible nor palpable), Grade 1: thyroid palpable but not visible, Grade 2: thyroid visible with neck in normal position. The total Goiter Rate (TGR) was calculated as the sum of goiter grade 1 and grade 2. To minimize error during data entry, double data entry was implemented.

\subsection{Data Processing and Analysis}

The filled questionnaires were checked for completeness and entered into EpiData 3.1 statistical software and then exported to SPSS version 20 for further analysis. Descriptive statistics were used to describe the study population in relation to relevant variables. Both bivariate and multivariate logistic regression models were used to identify associated factors. To minimize factors in multivariate analysis, only variables that showed a $p$-value $\leq 0.25$ on the bivariate analysis were entered into the multiple binary logistic regression models.

To see the linear correlation among the independent variables, multicollinearity was checked using standard error (SE). Variables with a standard error of $\geq 2$ were dropped from the multivariable analysis. The fitness of the model was tested by Hosmer-Lemeshow's goodness-of-fit test and Omnibus tests. The coefficient of fit was found to be insignificant with a large P-value, and the Omnibus tests were significant. Odds ratios with $95 \%$ confidence intervals (CIs) were computed, and variables with p-value less than 0.05 in multivariate analysis were considered statistically significant.

\subsection{Ethics Approval and Consent to Participate}


The study protocol was approved by Haramaya University, College of Health Ethical Review Board Committee (ERBC). The official letter of cooperation was written from Haramaya University, College of Health Science, to the study area. Before informed consent was obtained from each study participant, a clear description of the study title, purpose, procedures, durations, possible risks and benefits of the study was explained. The rights and privacy of the study participants during the interview were guaranteed in advance and asked in a private room. For those whose age was less than 18 years old, assent form from them and consent from their parent or husband was taken, and the responses of interviewees were kept confidential. Then, written and signed informed consent was obtained from each respondent before starting the interview. All questionnaires were coded with numbers to maintain the confidentiality of information gathered from each study participant throughout the study. Written informed consent was obtained from each participant.

\section{Results}

\subsection{Sociodemographic Characteristics of Study Participants}

Out of 584 respondents expected to participate in the study, 582 respondents were interviewed, resulting in a response rate of $99.6 \%$. The mean age $\pm \mathrm{SD}$ of the respondents was $30.49 \pm 7.78$ years. The majority of the respondents $500(86 \%)$ were Goffa by ethnicity, half 295 (50.5\%) were protestant by religion, 193 (33.3\%) were without formal education, 446 (76.4\%) were married and 335 (57.5\%) were housewives by occupation. Out of 446 married respondents, (37.4\%) and (58.5\%) respondent's husbands were without formal education and farmer, respectively. Approximately $56 \%$ of respondents had a family size greater than five (Table 1).

Table 1. Sociodemographic characteristics among women of reproductive age in Demba Goffa Woreda Southern Ethiopia, March 2016.

\footnotetext{
Variables

Frequency

Percentage (\%) 


\begin{tabular}{|c|c|c|}
\hline Variables & Frequency & Percentage (\%) \\
\hline $15-24$ & 150 & 25.8 \\
\hline $25-34$ & 244 & 41.9 \\
\hline$\geq 35$ & 188 & 32.3 \\
\hline \multicolumn{3}{|l|}{ Religion } \\
\hline Protestant & 295 & 50.7 \\
\hline Orthodox & 215 & 36.9 \\
\hline Muslim & 72 & 12.4 \\
\hline \multicolumn{3}{|l|}{ Ethnicity } \\
\hline Goffa & 500 & 86 \\
\hline Gamo & 62 & 10.6 \\
\hline Others* & 20 & 3.4 \\
\hline \multicolumn{3}{|l|}{ Marital status } \\
\hline Single & 95 & 16.3 \\
\hline Married & 446 & 76.6 \\
\hline Divorced & 41 & 7.1 \\
\hline \multicolumn{3}{|c|}{ Educational status of respondents } \\
\hline Without formal education & 193 & 33.3 \\
\hline Read and write & 115 & 19.7 \\
\hline Elementary school & 202 & 34.6 \\
\hline High school and above & 72 & 12.3 \\
\hline \multicolumn{3}{|l|}{ Occupation of respondent } \\
\hline Housewife & 335 & 57.5 \\
\hline Government employer & 55 & 9.4 \\
\hline Self-employer & 104 & 17.8 \\
\hline Student & 88 & 15.2 \\
\hline
\end{tabular}




\begin{tabular}{lll}
\hline Variables & Frequency & Percentage (\%) \\
\hline Educational status of husband & 167 & 37.4 \\
Illiterate & 51 & 11.4 \\
Read and write & 120 & 26.9 \\
Elementary school & 108 & 24.2 \\
High school and above & & \\
Occupation of husband & 261 & 58.5 \\
Farmer & 90 & 20.2 \\
Merchant & 82 & 18.4 \\
Government Employer & 11 & 2.5 \\
Others** & & \\
Family size & 256 & 44.1 \\
$\leq 5$ & 326 & 55.9 \\
$>5$ & & \\
\hline
\end{tabular}

Others* Wolayta, Gurage and Amhara

Others** Student and daily laborer

\subsection{Dietary Characteristics and Familial History of Goiter of Respondents}

The majority of the respondents $462(79.2 \%)$ eat fish/milk/meat, of these, $113(24 \%)$ eat three times and above per week. More than three-fourths of the respondents 444 (76\%) consumed cassava, of whom 340 (76\%) were eating three times and above per week. Of 485 (85\%) cabbage consumers, more than fourfifths of 402 (83) consumed cabbage less than three times per week. More than three-fifths of respondents $356(61.2 \%)$ used coarse non-packed salt, and only on-third (36.5\%) of these respondents used packed iodized salt. Of those who used packed iodized salt, most (99.5\%) used salt for less than six months. The majority of the respondents 499 (85.7\%) had no familial history of goiter (Table 2).

Table 2. Datary characteristics among women of reproductive age in Demba Goffa Woreda Southern 
Ethiopia, March 2016.

\begin{tabular}{|c|c|c|}
\hline Variables & Total frequency & $\overline{\text { Percent }(\%)}$ \\
\hline \multicolumn{3}{|l|}{ Eating fish/meat/milk } \\
\hline Yes & 462 & 79.2 \\
\hline No & 121 & 20.8 \\
\hline \multicolumn{3}{|c|}{ Frequency of eating fish $/$ meat $/$ milk } \\
\hline$<3$ times/week & 350 & 75.6 \\
\hline$\geq 3$ times/week & 112 & 24.4 \\
\hline \multicolumn{3}{|l|}{ Eating cassava } \\
\hline Yes & 444 & 76.2 \\
\hline No & 138 & 23.8 \\
\hline \multicolumn{3}{|c|}{ Frequency of eating cassava } \\
\hline$<3$ times/week & 340 & 76.6 \\
\hline$\geq 3$ times/week & 104 & 23.4 \\
\hline \multicolumn{3}{|l|}{ Eating Cabbage } \\
\hline Yes & 485 & 83 \\
\hline No & 97 & 17 \\
\hline \multicolumn{3}{|c|}{ Frequency of eating cabbage } \\
\hline$<3$ times/week & 402 & 83 \\
\hline$\geq 3$ times/week & 83 & 17 \\
\hline \multicolumn{3}{|l|}{ Type of salt used } \\
\hline Packed iodized salt & 226 & 38.8 \\
\hline Non packed (coarse salt) & 356 & 61.2 \\
\hline \multicolumn{3}{|c|}{ Familial History of Goiter } \\
\hline Have & 83 & 14.3 \\
\hline
\end{tabular}




\subsection{Knowledge of IDD of Respondents}

In this study, more than three quarters of respondents (71.6\%) had poor knowledge of Iodized salt and IDD.

\subsection{Practice of Iodized Salt Utilization}

Of the total respondents, most of them 552 (95\%) keep the salt in dry areas, but only one-third $190(32.5 \%)$ of respondents expose the salt to sun when the salt becomes humid. More than half of the respondents 361 (62\%) put the salt in a container having cover, but only $120(19 \%)$ wash the salt before use. More than three-fourths of the participants, $399(68.6 \%)$ used salt for $\leq 2$ months once purchased. More than a half $331(56.9 \%)$ add salt at the end when cooking food, and only $160(27.5 \%)$ have information about iodized salt. Of them, 97 (60.8\%) receive the information from health professionals (Table 3).

Table 3. Knowledge and practice iodized salt characteristics among women of reproductive age in Demba Goffa Woreda, Southern Ethiopia, March, 2016.

Variables

\section{Place of keeping}

Dry area

Moist and near to fire

\section{How to keep salt}

Covered

Open

\section{Sun expose}

Yes

No

\section{Salt wash}

Yes

\section{Frequency}

Percentage (\%)

552

95

30

5

221

38 
When to use salt

At the end

While cooking

How long salt kept

$<=2$ months

$>2$ month

Information about iodized salt

Yes

160

No

Knowledge towards prevention of goiter and IDD

Poor

Good

Source of information about Iodized salt

Health professionals

97

46

17
71.8

60.6

29

10.4

Others*** Television and relativities

\subsection{Prevalence of Goiter among Women}

The total goiter rate was $43.3 \%(95 \% \mathrm{CI}=39.2,46.9)$, of which $32 \%$ was palpable and $11 \%$ was visible. The goiter rate was higher in the 24-34 age group (16.6\%) (Figure 1). 


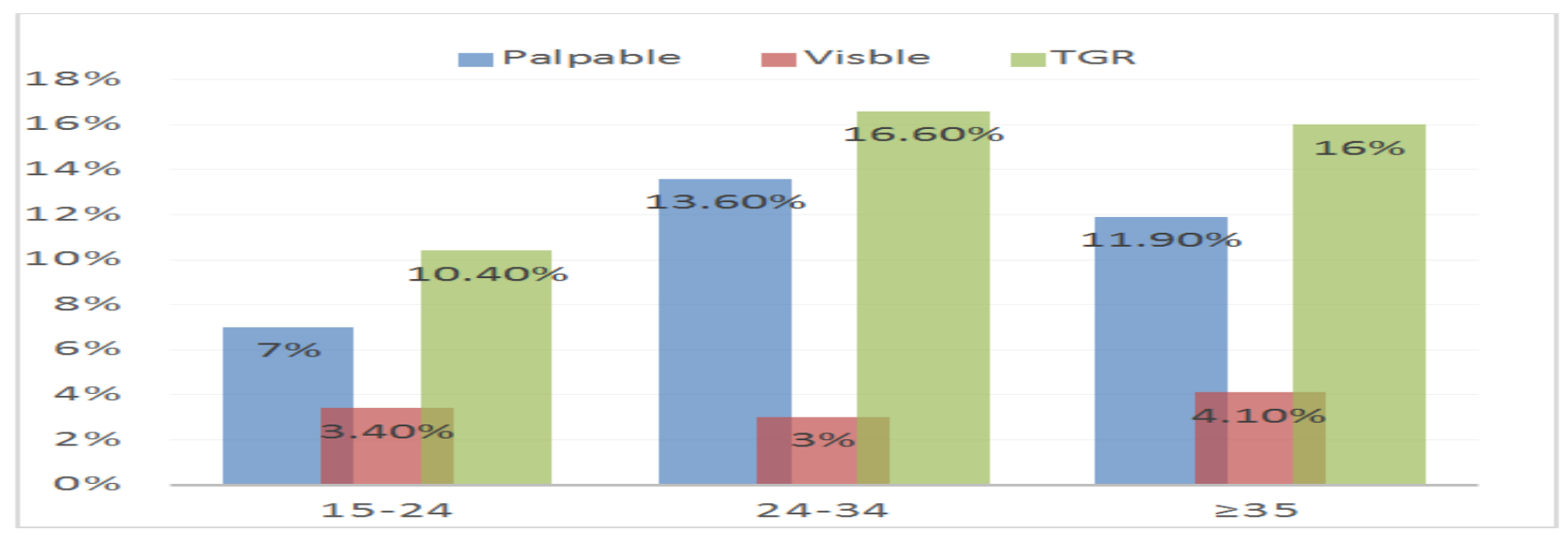

Figure 1. Prevalence of goiter in respondents by age category women of reproductive age in Demba Goffa Woreda, Southern Ethiopia, 2016.

\subsection{Factors Associated with Goiter}

In the bivariate logistic regression analysis, family size, fish/meat/milk consumption, cassava consumption, cabbage consumption frequency, family history of goiter, type of salt used, how the salt was stored, exposing the salt to sun, washing the salt before use, period of salt usage once purchased, the time to add the salt to the food and knowledge towards IDD were significantly associated with goiter (Table 4). In the multivariate logistic regression analysis, income, cassava consumption, family history of goiter, salt wash before use, period of salt usage once purchased and knowledge about IDD were significantly associated with goiter. Participants whose income $<500$ ETB were four times (AOR: 4.2, 95\% CI: 1.7-10) more likely to have goiter than those with income $\geq 999$ (Table 4).

Table 4. Results of multivariate logistic regression analysis of factors independently associated with goiter among women of reproductive age in Demba Goffa Woreda, Southern Ethiopia, 2016.

\begin{tabular}{|c|c|c|c|c|}
\hline \multirow{2}{*}{ Variables } & \multicolumn{2}{|l|}{ Goiter } & \multirow{2}{*}{$\begin{array}{l}\text { Crude } \\
(95 \% \mathrm{CI})\end{array}$} & \multirow{2}{*}{ Adjusted OR (95\%CI) } \\
\hline & Yes $(\%)$ & No $(\%)$ & & \\
\hline \multicolumn{5}{|c|}{ Family size } \\
\hline$<5$ & $87(33.8)$ & $170(76.2)$ & 1.00 & 1.00 \\
\hline$>=5$ & $176(54.6)$ & $149(45.4)$ & $2.3(1.6-3.2)$ & $1.64(0.65-2.24)$ \\
\hline \multicolumn{5}{|c|}{ Eating fish/meat/milk } \\
\hline Yes & $144(70.2)$ & $205(29.8)$ & 1.00 & 1.00 \\
\hline
\end{tabular}




\begin{tabular}{lllll}
\hline No & $38(27.5)$ & $93(82.3)$ & $3.3(1.9-5.5)$ & $1.1(0.8-2.1)$
\end{tabular}

\section{Eating cassava}

$\begin{array}{lllll}\text { Yes } & 225(49.3) & 219(50.7) & 2.7(1.7-4) & \mathbf{2 . 0 2}(\mathbf{1 . 0 3 - 4}) * \\ \text { No } & 38(27.5) & 100(72.5) & 1.00 & 1.00\end{array}$

\section{Cabbage frequency}

$<3$ times/week

$168(41.8)$

$234(59.2)$

1.00

1.00

$>=3$ times/week

$64(77) \quad 19(23)$

$4.7(2.7-8.1)$

$0.96(0.38-2.45)$

\section{Family history of goiter}

$\begin{array}{lllll}\text { Have } & 73(87.9) & 10(12.1 & 11.8(5.9-23.5) & \mathbf{4 . 6 ( 1 . 4 - 1 5 . 8 ) *} \\ \text { Have no } & 190(38.1) & 309(61.9) & 1.00 & 1.00\end{array}$

\section{Type of salt}

Packed iodized

$70(30.9) \quad 156(69.1) \quad 1.00$

1.00

Non packed coarse

193 (54.2)

163 (45.8)

$2.6(1.85-3.75)$

$1.6(0.8-3.2)$

\section{How do you keep salt}

Closed container $99(27.4) \quad 262(72.6)$

1.00

1.00

Open container

$164(74.2)$

57 (25.8)

$7.6(5.2-11.1)$

$0.5(0.3-1.1)$

\section{Sun expose of salt}

Yes

$158(83.2)$

No

105 (26.8)

32 (16.8)

13.5 (8.7-20.9)

$1.2(0.5-2.80$

Wash salt

Yes

$$
108(94.7) \quad 6(5.3)
$$

$36(15.6-84.6)$

1.00

No

155 (31.9) $313(68.1)$

1.00

3.4 (1.1-11.3)*

1.00

How long salt use once purchased

$<=2$ month

$91(22.8)$

308 (87.2)

1.00

1.00

$>2$ month.

$172(93.9)$

$11(6.1)$

$53(27.5-102)$

$11(5-26) * *$

When to use salt cooking food

$\begin{array}{lllll}\text { At the end } & 78(23.6) & 253(76.4) & 1.00 & 1.00 \\ \text { While cooking } & 185(81) & 43(19) & 9.1(6.2-13.3) & 1.32(0.8-2.12)\end{array}$

\section{Knowledge towards IDD}

$\begin{array}{lllll}\text { Good } & 204(49.3) & 210(50.7) & 1.00 & 1.00 \\ \text { Poor } & 59(64.9) & 109(35.1) & 1.79(1.23-2.6) & \mathbf{2 . 7}(\mathbf{1 . 4 - 5 . 5}) *\end{array}$

Respondents who had consumed cassava were 2 times (AOR: 2.02, 95\% CI: 3.6-881.03-4) more likely to have goiter than their counterparts. Family history of goiter also found a significant association with goiter. 
Respondents who had a family history of goiter were 4.6 times (AOR: 4.6, 95\% CI: 1.4-15.8) more likely to have goiter than those had no family history of goiter. Participants who washed the salt before use were more than three times (AOR: 3.4. 95\% CI: 1.1-11.3) more likely to have goiter than those not wash the salt. The period of use salt at household once purchased was found to be a strong significant factor for goiter. Those who used the salt for more than two months were nearly twelve times (AOR: 11, 95\% CI: 5-26) more likely to have goiter compared to their counterparts who used $\leq 2$ months.

Knowledge about IDDs was also found to have a significant association with goiter. Participants who had poor knowledge about IDD were nearly three times (AOR: 2.7, 95\% CI: 1.4-5.5) more likely to have goiter than their counterparts (Table 4) 


\section{Discussion}

The study revealed that the TGP was $43.3 \%$ (95\% CI: 39.2, 46.9), of which $32 \%$ were palpable and $11 \%$ were visible goiters. Cassava consumption, family history of goiter, salt wash before use, period of salt usage once purchased and knowledge towards IDD were significantly associated with goiter.

The prevalence of this study TGR was found to be higher than the national TGR, which was $35.8 \%$ (24.3\% palpable and $11.5 \%$ visible) [5]. It was also higher than a study done in West Gojjam, which was $30.1 \%$ [15]. However, the current finding is below the regional state in Ethiopia TGR, which was $60 \%$ (43\% palpable and $18 \%$ visible) [5], and a higher prevalence was found in the 25-34 age group. However, a study performed in Pakistan showed that the highest prevalence was found in the younger age group [16]. The increased rate of goiter in the current study might be due to Gamo Goffa being an endemic area for goiter, and the nutritional condition of the study population is quite different from other studies. The possible reason behind the discrepancy in age group might be related to the decrease in the prevalence of goiter as the age of these populations' increases.

A longer (more than 2 months) storage time of salt at household once purchased was strongly associated with goiter. A study performed in Maychew District also showed that when the period of stay of salt at household increases once purchased, the adequacy of iodine in the salt will decrease [17]. A similar study conducted in Colombia showed that the effect of longer storage beyond 2 months aggravated losses of iodine from the salt [18]. Another study performed in Canada also strengthened this idea, with salt losses of $28 \%-51 \%$ and $35 \%-52 \%$ of its iodine content after three months and six months, respectively [19]. This is because when the salt stored for a longer time, as iodine is an easily volatile element, iodine found in the salt will be easily loosened, and finally, the salts become inadequately iodized, which may result in goiter. On the other hand, due to different environmental conditions during storage and distribution, the quality of iodine may be affected. 
Cassava consumption was identified to be significantly associated with goiter. Respondents who consumed cassava were more likely to have goiter than their counterparts who did not consumed cassava. A similar study done at Sawla also showed that cassava consumption is a risk factor for goiter [20]. Likewise, a study conducted in five regions of Ethiopia in 2008 also showed that cassava consumption is a risk factor for IDD. In the two regions SNNP and Benshangul Gumuz, those who consumed cassava frequently were significantly affected by goiter than those who consume cassava rarely or not [21]. This is because cassava contains goitrogens such as thiocyanate and isothiocyanate that inhibit the uptake of iodine to thyroid follicular cells and block the thyroid peroxidase enzyme. In the presence of goitrogens, iodination of thyroglobulin protein will be affected, inducing poor thyroxine production.

Knowledge towards iodized salt and prevention of IDD was significantly associated with goiter. This study was consistent with the study done in Gondar [17], Mali [21] and Ghana [3]. This is because when the knowledge of participants towards the use of iodized salt is increased, they will have a good understanding of how to prevent IDD which was directly associated with the availability of adequate salt for all members of the household.

Salt wash before use was significantly associated with goiter. This finding is in agreement with the study done in Gondar Town [17]. This is because as iodine is an easily volatile element, and when the salt is washed, the iodine in the salt will be easily loosened and result in inadequate of iodine in the salt, finally this result in goiter.

Family history of goiter was also found to be significantly associated with goiter. Respondents who had a family history were more likely to have goiter than those who had no family history of goiter [17]. This may be due to respondents having a family history of goiter and their families were living together and having similar dietary habits. Another reason may be that iodine deficiency is an intergenerational, meaning that the deficiency passes through generation. 
This finding will support the strategic objectives of the National Nutrition Program II (NNP-II), and Food and Nutrition Policy (FNP) in Ethiopia, which was launched to improve the nutritional status of women of reproductive age, including adolescent girls, pregnant women, and lactating mothers, through appropriate nutrition to alleviate the current burden of micronutrient deficiencies like IDD. In this study, consumption of cassava is significantly associated with goiter. It also supported by the existed evidence. Therefore, based on this finding, it is recommended that avoiding and minimizing frequent consumption of cassava.

Regarding the limitations of the study, this study is limited because do not use ultrasound that the best way to assess goiter is ultrasound. Urinary iodine level is also better than assessing only by history and physical examination, as it may result inters observer variation. However, the limitation was minimized by intensive training on goiter examination by medical doctors. The other limitation of this study is recall bias, which was minimized by probing the study participants.

\section{Conclusion}

Iodine deficiency was found to be a severe problem in women of reproductive age. Cassava consumption, family history of goiter, salt wash before use, period of salt usage once purchased and knowledge of IDD were significantly associated with goiter.

Thus, urgent supplementation with iodine and improved access to foods rich in iodine and intake of iodized salt by nearby health facilities and health bureau is highly recommended. Health education on the importance of iodized salt and its proper usage, and the effect of goitrogenic foods on the thyroid gland should be given by nearby health facilities and health bureau. Because IDD is the major public health problem in the country and still the prevalence is high in the study area, sectors of governmental, non-governmental and concerned bodies near the study area should work collaborate to control or minimize the problem. Further studies should be conducted using larger sample sizes and biomarkers to verify factors influencing iodine deficiency disorder in Demba 
Goffa Woreda communities.

\section{Data Availability}

The data used to support the findings of this study are available from the corresponding author upon reasonable request.

\section{Disclosure}

This manuscript was prepared from the thesis work of Mrs. Befikaduwa Zekarias. The cost of conducting this research was covered by her.

\section{Authors' Contributions}

BZ: involved in the design, selection of articles, statistical analysis and manuscript writing. FM, $\mathrm{BM}$, AT and LG assisted in the analysis, manuscript preparation, editing and intellectual contribution. All authors read and approved the final draft of the manuscript.

\section{Conflict of Interests}

The authors declare that they have no conflicts of interest in this work.

\section{Acknowledgements}

We express our special thanks to all study participants who took part in the study, data collectors and supervisors who had marvelous contributions to the success of the study.

\section{References}

[1] Ogbera, A. O. and O. E. Okisieme. Thyroid disease in Africa: Epidemiology and Management Challenges. Thyroid International. 2014.

[2] Zenebe Negeri, Teshome Gobena, et al. Determining the Magnitude of Iodine Deficiency and Its Associated Risk Factors among Pregnant Women Visiting Jimma University Specialized Hospital for Antenatal Care. World Journal of Medicine and Medical Science. 2014. 2 (4): PP: 1-16.

[3] Bruno de B, Maria A, et al. Iodine status worldwide. WHO Global IDD database on iodine deficiency. WHO Geneva. 2004.

[4] CSA (Central statistical Agency), Ethiopia Demographic and Health Survey. 2016. Addis Ababa, Ethiopia.

[5] Chernet Abuye, Yemane Berhane, et al The role of changing diet and altitude on goitre prevalence in five regional states in Ethiopia. East Afrikan jornal of Public Health. 2008. 5: 163-168. 
[6] Torheim LE, Granli GI, et al. Women's iodine status and its determinants in an iodine-deficient area in the Kayes region, Mali. Public Health Nutr. 2005. 8: 387-394.

[7] Bruno de B, Maria A, et al. Iodine status worldwide. WHO Global IDD database on iodine deficiency. WHO Geneva. 2004.

[8] Kousar, J. and Z. F. Kawaoosa. To estimate the magnitude goiter among women in reproductive age group (15 -49 years) in the Kashmir population. Journal of community Nutrition and Health. 2013. 2(1).

[9] Qamar, M. F., et al. Prevalence and case reports of goiter at district Bahawalpur, Pakistan. 2012. 24(1): ISSN 1013 - 5316.

[10]Ogbera, A. O. and O. E. Okisieme. Thyroid disease in Africa: Epidemiology and Management Challenges._Thyroid International. 2014.

[11] Joste. P. and M. Andersson. Iodine Nutrition in Africa: Sight and life. 2014. 27(3).

[12] Chernet Abuye and Yemane Berhane. The goiter rate, its association with reproductive failure, and the knowledge of iodine deficiency disorders (IDD) among women in Ethiopia. BMC Public Health. 2007. 7 (316): 1-7

[13]B. Strange, M. Joseph, et al. Reaching the rural poor in India with iodized salt: the Micronutrient Initiative's Iodized Salt Coverage DD. News Letter. 2011. 39, 6-8.

[14]Tafere Gebreegziabher, Nega Teyike, et al. Lack of dietary sources of iodine and the prevalence of iodine deficiency in rural women from Sidama Zone, Southern Ethiopia. 2013.5 (13).

[15]Haji Kedir, Yemane Berhane, et al. Subclinical Iodine Deficiency among Pregnant Women in Haramaya District, Eastern Ethiopia. Journal of Nutrition and Metabolism. 2014.

[16] Mousavi SM, Tavakisk factooli N, et al. Risk factors for goiter in primary school girls in Qom city of Iran. European Journal of Clinical Nutrition. 2006. (60): 426-433.

[17] Hailay Gebrearegawi, Melkie Edris, Yesuf, et al. Availability of Adequately Iodized Salt at Household Level and Associated Factors in Gondar Town, Northwest Ethiopia. ISRN Public Health. 2013. 6.

[18] Gidey B, Alemu K, et al. Availability of Adequate Iodized Salt at Household Level and Associated Factors in Rural Communities in Laelay Maychew District, Northern Ethiopia. Journal of Nutrition and Health Sciences. 2015. 2 (ISSN: 2393-9060).

[19] Diosady L, Alberti J, et al. Stability of iodine in iodine salt used for correction of iodine deficiency disorder. Food and Nutrition Bulten. 1997. 18: 388-96.

[20] World Health Organization (WHO). Iodine Deficiency Disorders. Global Database. 2013.

[21] M. B. Zimmermann. The effects of iodine deficiency in pregnancy and infancy. Journal of Pediatrics and Perinatal Epidemiology. 2012. 26: 108-117. 
[22] Deresse Legesse Kebede and Yohannes Mehretie Adinew. Predictors of Goiter among School Children in Southwest Ethiopia. Journal of Nutrition and Food Science. 2015. 5:3. 
Figures

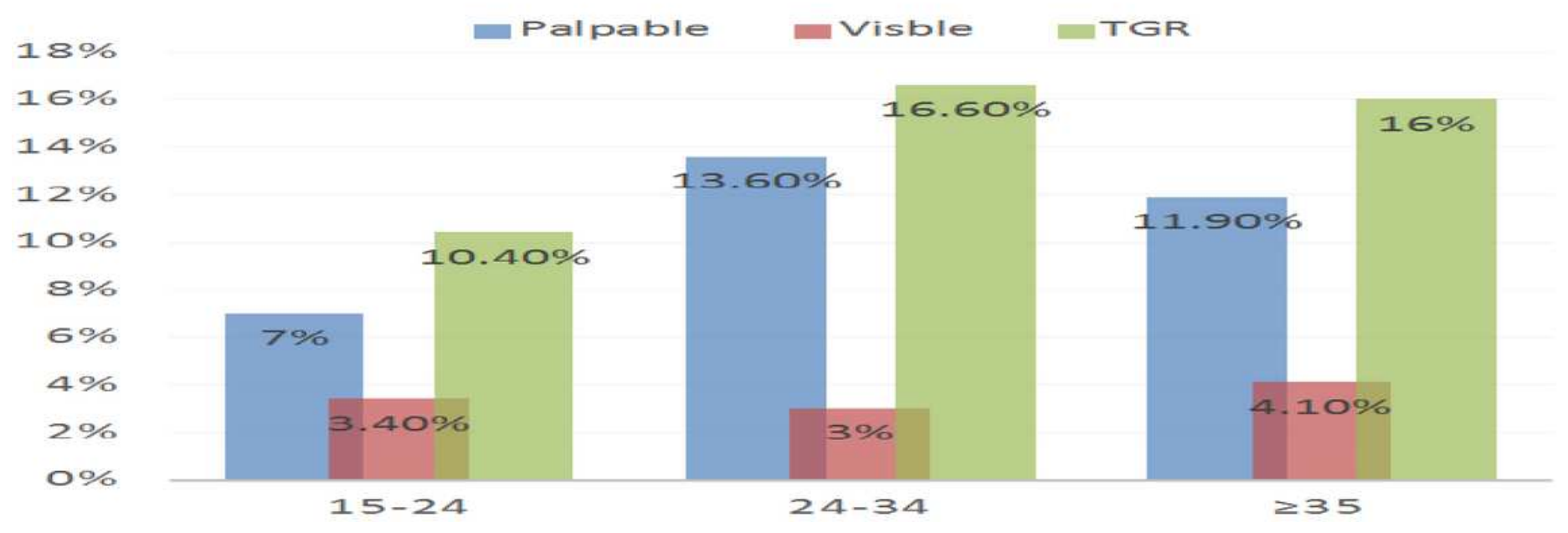

Figure 1

Prevalence of goiter in respondents by age category women of reproductive age in Demba Goffa Woreda, Southern Ethiopia, 2016. 